\title{
Enfermedad diarreica por rotavirus en brotes epidémicos
}

\author{
Jesús Reyna-Figueroa, ${ }^{1}$ Edgar Sánchez-Uribe, ${ }^{1}$ \\ Alejandra Esteves-Jaramillo, ${ }^{1}$ Luz del Carmen Hernández-Hernández ${ }^{1}$ \\ y Vesta Richardson ${ }^{1}$
}

Forma de citar

Reyna-Figueroa J, Sánchez-Uribe E, Esteves-Jaramillo A, Hernández-Hernández LC, Richardson V. Enfermedad diarreica por rotavirus en brotes epidémicos. Rev Panam Salud Publica. 2012;31(2):142-7.

RESUMEN Objetivo. Determinar el perfil epidemiológico de los brotes de enfermedad diarreica aguda por rotavirus $(R V)$ ocurridos en pacientes pediátricos, mediante una revisión crítica de la literatura publicada entre 2000 y 2010.

Métodos. Se realizó una búsqueda de artículos publicados desde enero de 2000 hasta abril de 2010, recogidos por las bases de datos Artemisa, EBSCO, Embase, Imbiomed, Lilacs, Ovid, PubMed y Science Direct. En los estudios que cumplieron con los criterios de inclusión, se identificaron posibles factores de confusión y se atribuyeron riesgos de sesgo con base en el número de ítems considerados inadecuados en cada caso. Se describieron las características epidemiológicas y microbiológicas de los brotes.

Resultados. Solo $14(10,8 \%)$ de los 129 títulos identificados formaron parte de la muestra, los cuales sumaron 91092 casos de diarrea aguda notificados. En 5250 de estos casos se realizó la búsqueda de RV, la cual arrojó 1711 (32,5\%) aislamientos positivos. Se observó que el $R V$ del grupo A fue el agente causal en 100\% de los brotes, mientras que el genotipo G9 fue documentado en $50 \%$ de los artículos.

Conclusiones. El RV, principalmente el serotipo G9, fue uno de los principales agentes responsables de los brotes de EDA en la última década. Un cuidadoso estudio de brote puede aportar información valiosa para el control y la prevención de la enfermedad por $R V$.

Palabras clave Rotavirus; diarrea infantil; salud del niño; enfermedades gastrointestinales; brotes de enfermedades.

La enfermedad diarreica aguda (EDA) es la segunda causa de muerte en menores de 5 años y se ubica como uno de los principales problemas de salud pública en todo el mundo (1). El descubrimiento de rotavirus $(\mathrm{RV})$ en los años setenta supuso un escenario de tranquilidad, ya

\footnotetext{
Centro Nacional para la Salud de la Infancia y la Adolescencia, Unidad de Investigación, México D.F., México. La correspondencia se debe dirigir a Jesús Reyna-Figueroa. Correo electrónico: jesusreynaf@prodigy.net.mx
}

que la sintomatología asociada al nuevo agente causal se consideraba menos grave que la presentada por Salmonella, Vibrio cholerae, Shigella o Campylobacter. Sin embargo, ese panorama cambió con el paso de los años al establecerse el alto grado de transmisibilidad que entraña el RV, así como su participación en el incremento en las tasas de mortalidad por $\operatorname{EDA}(2,3)$. Actualmente se reconoce que los genotipos G1P[8], G2P[4], G3P[8] y G4P[8] representan $90 \%$ del total de los aislamientos, mientras que genotipos como el G9P[8] y en menor medida G5, G8, G6 y otras combinaciones de G9 son considerados emergentes (4-6).

A pesar de las medidas que buscaron mejorar el saneamiento y disminuir la morbi-mortalidad por EDA, la infección por RV ha seguido causando un gran número de hospitalizaciones y defunciones $(7,8)$. Tal situación llevó a que a principios de los años ochenta se comenzaran a realizar investigaciones para desarrollar vacunas efectivas contra $\operatorname{RV}(9,10)$. Desde 1974, cuando se notificó el primer 
brote de EDA por RV, se han documentado epidemias de esta enfermedad que han tenido un gran impacto en la morbimortalidad infantil $(3,11,12)$.

Entre las medidas que contribuyen a controlar los brotes de EDA por RV se encuentran la notificación inmediata, la planificación de los recursos sanitarios, el estudio detallado de los factores de riesgo que contribuyen a su aparición, la información de las cepas circulantes y la evaluación de la efectividad vacunal. Todas repercuten de manera positiva en la eficacia de las estrategias de prevención existentes y arrojan lecciones que pueden prevenir brotes similares en el futuro $(3,12,13)$.

Por lo anterior, organismos a nivel internacional, tales como los Centros para el Control y la Prevención de Enfermedades (Estados Unidos) han dictado recomendaciones para la identificación y estudio correcto de un brote por EDA $(12,14,15)$.

El objetivo del presente trabajo es determinar el perfil epidemiológico de los brotes de EDA por RV ocurridos en pacientes pediátricos, mediante una revisión crítica de la literatura publicada entre 2000 y 2010.

\section{MÉTODOS}

Se revisaron estudios epidemiológicos descriptivos no comparativos, de casos y controles y de cohortes que describieron un brote de EDA por RV en una población determinada. ${ }^{2}$ En cada trabajo se hizo hincapié principalmente en metodología utilizada, grupo de edad afectado, densidad poblacional de la región estudiada, existencia $-\mathrm{O}$ no- de un sistema de vigilancia epidemiológica, número de casos de EDA notificados, número de casos muestreados, número de casos infectados con RV, serotipo o genotipo involucrado y riesgo de sesgo en los resultados.

Se realizó indagación bibliográfica en Artemisa, EBSCO, Embase, Imbiomed, Lilacs, Ovid, PubMed y Science Direct, incluyéndose los estudios publicados entre 2000 y 2010 en formato completo. Además, en cada uno de estos trabajos se revisó la sección de referencias con objeto de identificar otros estudios potencialmente relevantes para el análisis. Los términos clave utilizados para el idioma

\footnotetext{
2 No se incluyeron estudios de brotes intrahospitalarios.
}

inglés fueron "rotavirus outbreak", "rotavirus AND outbreak", "pediatrics rotavirus outbreak" y "diarrhea outbreak", mientras que en español fueron: "brote por rotavirus", "rotavirus AND brote", "brote de rotavirus AND niños" y "brote AND diarrea".

Los criterios de selección de los estudios que serían examinados incluyeron: i) que fueran realizados en poblaciones de entre 0 y 18 años de edad (en los casos en que describían niños y adultos, solo se consideraron los datos de los primeros, y si no discriminaban entre grupos de edad fueron excluidos), ii) que describieran brotes de EDA según su definición operativa o un incremento significativo de casos en relación con los valores esperados en la población estudiada, iii) que especificaran el lugar y la fecha del brote y iv) que incluyeran aislamientos microbiológicos de RV. Se seleccionaron un total de 14 artículos, de los cuales un mayor número correspondieron al período 2001-2005.

Utilizando el análisis de calidad como medio de interpretación de resultados, a cada estudio se le asignó un determinado riesgo de sesgo. Mediante la escala Newcastle-Ottawa para estudios no aleatorizados, se identificaron posibles factores de confusión y se atribuyeron riesgos de sesgo con base en el número de ítems considerados inadecuados en cada estudio (16). De este modo se establecieron tres niveles de riesgo:

- Bajo: cuando de trató de sesgos que, por referirse a medidas de resultado primarias, tienen pocas probabilidades de cambiar significativamente los resultados del estudio.

- Medio: en los casos en que un sesgo plausible planteaba dudas acerca de la validez de los resultados debido a que una o dos medidas de resultado primarias no se reportaban.

- Alto: cuando hubo un sesgo plausible que debilitaba significativamente la confianza en los resultados, por ejemplo si no se reportaban más de dos medidas de resultado primarias. Los estudios afectados con este tipo de sesgos fueron excluidos del análisis.

\section{RESULTADOS}

La búsqueda arrojó un total de 129 títulos, de los cuales 108 fueron excluidos porque no estudiaban brotes de EDA. De los 21 restantes, solo 14 trabajos cum- plieron con los criterios de inclusión y calidad. La principal fuente de sesgo fue la falta de información para completar cada una de las variables establecidas para este análisis (cuadro 1). Tres estudios fueron realizados en Australia y tres en la India, dos en Italia y uno en cada uno de los siguientes países: Albania, Canadá, El Salvador, Indonesia, Jamaica y Nicaragua (17-30) (cuadro 2). El número de casos de EDA reportados en todos los estudios totalizó 90 816, con un rango de 24 a 64000 casos. De los brotes incluidos, el grupo de edad con más prevalencia fue el de menores de 5 años- representando entre $12 \%$ y $97 \%$ de las poblaciones afectadas.

La mortalidad descrita en menores de 5 años de edad fue de $0,09 \%$ a $0,88 \%$, mientras que apenas 5 de los 14 estudios reportaron tasas de mortalidad por EDA $(20,22,25,26,28)$ y 8 utilizaron 2 ó más métodos para identificar RV (cuadro 2). El total de casos muestreados para análisis microbiológico fue de 2285 (2,51\%), de los cuales $934(40,8 \%)$ dieron positivo a RV. En los 14 estudios se identificó RV del grupo A y solo en 9 (60\%) se realizó genotipificación, hallándose G9P[8] en 4 (28,5\%), G1P[8] en $2(14,2 \%), \mathrm{G} 4 \mathrm{P}[8]$ en 1 $(7,2 \%)$, G9 en $1(7,2 \%)$ y G3 en $1(7,2 \%)$.

Cabe destacar que en ocho $(57 \%)$ de estos trabajos se realizó búsqueda de otros agentes infecciosos, encontrándose en dos de ellos coinfección con norovirus, astrovirus y adenovirus, mas no hubo coinfecciones bacterianas. Si bien $36 \%(5 / 14)$ de los brotes fueron generados por agua contaminada, solo en cuatro estudios se llevó a cabo un muestreo ambiental y en uno se documentó RV (cuadro 3).

\section{DISCUSIÓN}

La epidemiología de las infecciones por rotavirus es compleja. Si bien la morbilidad por RV puede presentarse en cualquier región del mundo, cerca de $82 \%$ de las muertes ocurren en países con bajo ingreso per cápita, mayor prevalencia de desnutrición y pobre acceso a los sistemas de salud $(37,38)$. Los casos detectados en humanos pertenecen en su mayoría al grupo A, y durante los últimos años una amplia gama de sus serotipos/genotipos han sido reconocidos como causantes de EDA, planteando un mayor reto diagnóstico por su gran tendencia al cambio. Por ejemplo, a partir de los años noventa el G9, 
CUADRO 1. Requisitos de inclusión aplicados a los estudios sobre brotes de enfermedad diarreica aguda (EDA) evaluados en el presente trabajo, 2000-2010

\begin{tabular}{|c|c|c|c|c|c|c|c|c|c|}
\hline Costa FF (5) & Descriptivo & No & NR & Sí & NR & $31 / 3$ & G9P(8) & Alto & No \\
\hline Snelling TL (17) & Casos y controles & Sí & 50000 & Sí & 186 & $171 / 78$ & G9P(8) & Bajo & Sí \\
\hline Gelbart B (18) & Descriptivo & Sí & 1000 & Sí & 24 & $14 / 8$ & Grupo A & Medio & Sí \\
\hline Karmakar S (21) & Descriptivo & Sí & 65000 & Sí & 1783 & $12 / 3$ & Grupo A & Medio & Sí \\
\hline Sugunan AP (22) & Descriptivo & Sí & 1346 & Sí & 113 & $20 / 19$ & Grupo A & Medio & Sí \\
\hline Martinelli D (23) & Casos y controles & Sí & NR & Sí & 2860 & $70 / 34$ & G9 & Medio & Sí \\
\hline Scarcella C (24) & Descriptivo & Sí & 3360 & Sí & 299 & $36 / 19$ & Grupo A & Medio & Sí \\
\hline Corwin AL (28) & Casos y controles & Sí & 236630 & Sí & 2600 & $27 / 13$ & Grupo A & Medio & Sí \\
\hline Villena C (29) & Descriptivo & Sí & NR & Sí & 1740 & $28 / 25$ & G9P(8) & Medio & Sí \\
\hline Ford-Jones EL (30) & Cohorte descriptiva & Sí & 816585 & Sí & 1638 & $1001 / 372$ & NR & Medio & Sí \\
\hline Amador $\mathrm{JJ}^{\mathrm{a}}(31)$ & Casos y controles & Sí & NR & Sí & 47470 & $67 / 28$ & G4 & Medio & No \\
\hline Lee Jl (32) & Descriptivo & Sí & NR & NR & NR & $3174 / 571$ & G3P(8) & Alto & No \\
\hline Krieg RE (33) & Descriptivo & Sí & NR & NR & 467 & $45 / 23$ & Grupo A & Alto & No \\
\hline Sum MS (34) & Descriptivo & No & NR & NR & NR & $272 / 75$ & G1P(8) & Alto & No \\
\hline Palombo EA (35) & Descriptivo & No & NR & Sí & 100 & No & G2 & Alto & No \\
\hline $\operatorname{CDC}(36)$ & Descriptivo & Sí & NR & Sí & NR & $43 / 21$ & NR & Alto & No \\
\hline
\end{tabular}

Fuente: elaboración de los autores.

Nota: NR: no referido en el estudio; CDC: (sigla en inglés de) Centros para el Control y la Prevención de Enfermedades (Estados Unidos)

a Este estudio se excluyó por describir el mismo brote en Nicaragua que Bucardo F et al. (23).

considerado como serotipo emergente, ha ganado relevancia a nivel mundial, aislándose en $4,1 \%$ del total infecciones por RV, y ha sido el más frecuentemente implicado en brotes de RV durante la última década $(4,39)$. La vacunación contra RV ha demostrado ser una estrategia de salud pública que, cuando es parte de un programa estructurado de aplicación, no solo reduce la mortalidad sino también los casos de enfermedad grave por este virus $(7,13)$. Sin embargo, la protección heterotípica que confiere en brotes deberá ser evaluada.

Vale señalar que el análisis de los resultados estuvo sujeto a algunas limitaciones, tales como las relacionadas con la heterogeneidad de los trabajos de la muestra en cuanto a diversidad geográfica, estacionalidad del brote, rango de edad, y estado nutricional y socioeconómico de las poblaciones estudiadas. Más aún, incluso cuando la mayor parte de los estudios presentó un sesgo medio que permitió realizar un análisis descriptivo adecuado, el análisis estadístico tuvo restricciones debidas a la diversidad en la manera en que se notificaron los datos.

En la mayoría de los artículos de la muestra no se evaluaron factores de gran protagonismo en la presentación de brotes de EDA, como la variabilidad regional donde se presentaron los eventos, la solidez de los sistemas de vigilancia epidemiológica, la propensión a desastres naturales y la infraestructura básica para prevenir esta enfermedad (p. ej. la calidad del agua y vacunación contra rotavirus), limitando el alcance de los análisis. Asimismo, en la mayoría de los trabajos revisados no se incluyeron como objetivos identificar la fuente de infección y determinar los principales factores de riesgo. No obstante, si bien muchos de los estudios concluyeron que la fuente del brote se relacionó a la contaminación del agua, ninguno realizó ponderaciones a través de medidas de asociación entre los probables factores que influyeron en la presentación del brote, limitándose a mencionar la presencia - en la mayoría de los casos- de hacinamiento, temporada invernal, situación socioeconómica baja, desnutrición, ausencia de acceso a agua de redes y falta de instalaciones para eliminación de excretas.

Aun cuando el presente trabajo no revisó estudios sobre brotes de EDA anteriores al período 2000-2010, se halló que la mayoría de las investigaciones sobre brotes de rotavirus fueron publicadas durante esta última década. Entre los factores que podrían haber contribuido a este mayor interés en el tema figuran i) un mayor acceso a técnicas diagnósticas para identificar el agente causal, ii) mejoras en la notificación y el registro de casos en los sistemas de vigilancia epi- 
CUADRO 2. Características epidemiológicas reportadas en los estudios sobre brotes de enfermedad diarreica aguda (EDA) evaluados en el presente trabajo, 2000-2010

\begin{tabular}{|c|c|c|c|c|c|c|}
\hline Kelkar SD (20) & India/Dic. 2000-Ene.2001 & $\begin{array}{l}\leq 6 \text { meses: } 15,3 \\
>6-12 \text { meses: } 61,2 \\
>12-24 \text { meses: } 18,4 \\
>24 \text { a } 60 \text { meses: } 5,3\end{array}$ & NR & 0,4 & NR & $\begin{array}{l}\text { Elisa, } \\
\text { PCR, } \\
\text { IgM en suero }\end{array}$ \\
\hline Villena C (29) & Albania/Dic. 2000-Ene. 2001 & $\begin{array}{l}0-5 \text { años: } 89,7 \\
6-9 \text { años: } 6,2 \\
10-15 \text { años: } 4,1\end{array}$ & NR & NR & NR & RT-PCR \\
\hline Guardado JA (26) & El Salvador/May. 2001-Abr.2002 & $\begin{array}{l}\text { (Solo para EDA por rotavirus) } \\
\leq 2 \text { años: } 97 \\
>2 \text { años: } 3\end{array}$ & 1,8 & 0,1 & 14 & Elisa \\
\hline Corwin AL (28) & Indonesia/Jun.-Ago. 2002 & $\begin{array}{l}<12 \text { meses: } 27,4 \\
11-60 \text { meses: } 40,7 \\
>60 \text { meses: } 31,8\end{array}$ & NR & 0,46 & 1,1 & $\begin{array}{l}\text { Elisa, } \\
\text { PCR }\end{array}$ \\
\hline Fischer TK (27) & Jamaica/2003 & NR & NR & NR & NR & $\begin{array}{l}\text { Elisa, aglutinación } \\
\text { en látex, RT-PCR, }\end{array}$ \\
\hline Sugunan AP (22) & India/Ene. 2004 & NR & NR & 0,88 & NR & $\begin{array}{l}\text { electroforesis de } \\
\text { ARN }\end{array}$ \\
\hline Bucardo F (25) & Nicaragua/Feb.-Mar. 2005 & $\begin{array}{l}<11 \text { meses: } 39,8 \\
12-23 \text { meses: } 26,8 \\
24-60 \text { meses: } 15,7 \\
>60 \text { meses: } 17,6\end{array}$ & NR & 0,09 & 0,12 & Elisa, PCR \\
\hline Gelbart B (18) & Australia/Sep.-Oct. 2005 & $\begin{array}{l}\text { < } 12 \text { meses: } 37,5 \\
12-23 \text { meses: } 20,8 \\
24-59 \text { meses: } 4,1 \\
>60 \text { meses: } 37,5\end{array}$ & NR & NR & NR & $\begin{array}{l}\text { Elisa o } \\
\text { inmunoensayo }\end{array}$ \\
\hline
\end{tabular}

Fuente: elaboración de los autores.

Nota: NR: no reportado en el estudio; PCR: (sigla en inglés de) reacción en cadena de la polimerasa; RT-PCR: (sigla en inglés de) reacción en cadena de la polimerasa con transcriptasa inversa; ARN: ácido ribonucleico. La suma de los porcentajes pueden no dar 100 debido al redondeo.

CUADRO 3. Muestra y resultado microbiológico en los estudios sobre brotes de enfermedad diarreica aguda evaluados en el presente trabajo que incluyeron examen ecológico, 2000-2010

\begin{tabular}{|c|c|c|}
\hline Autor (referencia) & Fuente de muestreo & Aislamiento microbiológico \\
\hline Karmakar S (21) & $\begin{array}{l}15 \text { muestras de agua de grifo, tubería, corriente, mineral embotellada, } \\
\text { almacenada y de manantial }\end{array}$ & $\begin{array}{l}80 \%(n=12) \text { muestras con aislamiento de coliformes: Klebsiella, } \\
\text { Escherichia coli, Aeromonas hydrophila, Citrobacter, Proteus }\end{array}$ \\
\hline Sugunan AP (22) & 5 muestras de agua de pozo & Todas ellas positivas a coliformes \\
\hline Martinelli D (23) & 70 muestras de agua de grifo y de mar, y mariscos & $\begin{array}{l}22,8 \%(n=16) \text { de muestras positivas: rotavirus }(15,7 \%) \text {, norovirus } \\
(5,7 \%) \text {, rotavirus + norovirus }(1,4 \%)\end{array}$ \\
\hline Scarcella C (24) & 94 muestras de agua de lago & $46,8 \%(n=44)$ muestras positivas: enterovirus y norovirus \\
\hline
\end{tabular}

Fuente: elaboración de los autores. 
demiológicos y iii) mayor accesibilidad a los sistemas de salud $(2,3,15,40,41)$.

Aparte de los estudios que no se incluyeron en el presente trabajo por no cumplir con los criterios de selección establecidos, seguramente hay otros que no fueron detectados durante la búsqueda, aun cuando se hizo lo posible por evitar este sesgo mediante el uso de buscadores reconocidos por la calidad de la información que manejan.

A pesar de sus limitaciones, este estudio resalta la participación de rotavirus en los brotes de EDA a nivel mundial, siendo el serotipo G9 el más frecuentemente aislado en los brotes de la última década. Reforzar la vigilancia epidemiológica activa, identificar los factores de riesgo que contribuyen a la aparición de la enfermedad, fortalecer las medidas de higiene y saneamiento e incorporar la vacunación contra RV a los programas de inmunización podrían lograr la reducción de la carga de esta enfermedad, principalmente en niños menores de 5 años.

\section{REFERENCIAS}

1. Bryce J, Boschi-Pinto C, Shibuya K, Black RE. WHO Child Health Epidemiology Reference Group. WHO estimates of the causes of death in children. Lancet. 2005;365(9465):1147-52.

2. Parashar UD, Gibson CJ, Bresee JS, Glass RI. Rotavirus and severe childhood diarrhea. Emerg Infect Dis. 2006;12(2):304-6.

3. Pan American Health Organization. Epidemiological surveillance of diarrheal diseases due to rotavirus: Field guide. Washington, D.C.: PAHO; 2010. (Scientific and Technical Publication No. 623).

4. Gentsch JR, Woods PA, Ramachandran M, Das BK, Leite JP, Alfieri A, et al. Review of $G$ and $P$ typing results from a global collection of rotavirus strains: implications for vaccine development. J Infect Dis. 1996;174 (1 supl):30-6.

5. Costa FF, Luchs A, Cilli A, Morillo SG, Carmona Rde C, Timenetsky Mdo C. Rotavirus genotype G9P[8] among Peguao Ty Indian community in São Paulo, Brazil. J Clin Virol. 2009;46(4):387-8.

6. Clark HF, Lawley DA, Schaffer A, Patacsil JM, Marcello AE, Glass RI, et al. Assessment of the epidemic potential of a new strain of rotavirus associated with the novel G9 serotype which caused an outbreak in the United States for the first time in the 1995-1996 season. J Clin Microbiol. 2004;42(4):1434-8.

7. Richardson V, Hernandez-Pichardo J, Quintanar-Solares M, Esparza-Aguilar M, Johnson B, Gomez-Altamirano CM, et al. Effect of rotavirus vaccination on death from childhood diarrhea in Mexico. N Engl J Med. 2010;362(4):299-305.

8. Velázquez FR, García-Lozano H, Rodríguez E, Cervantes Y, Gómez A, Melo M, Anaya $\mathrm{L}$, et al. Diarrhea morbidity and mortality in Mexican children: impact of rotavirus disease. Pediatr Infect Dis J. 2004;23(10 supl):149-55.

9. Desselberger U, Manktelow E, Li W, Cheung W, Iturriza-Gómara M, Gray J. Rotaviruses and rotavirus vaccines. Br Med Bull. 2009;90: 37-51.

10. Dennehy PH. Rotavirus vaccines: an overview. Clin Microbiol Rev. 2008;21(1):198-208.

11. Morris CA, Flewett TH, Bryden AS, Davies $H$. Epidemic viral enteritis in a long-stay children's ward. Lancet. 1975;1(7897):4-5

12. LeBaron CW, Furutan NP, Lew JF, Allen JR, Gouvea V, Moe C, et al. Viral agents of gastro- enteritis. Public health importance and outbreak management. MMWR Recomm Rep. 1990;39(RR-5):1-24.

13. Esparza-Aguilar M, Bautista-Márquez A, González-Andrade MC, Richardson-LópezCollada VL. Mortalidad por enfermedad diarreica en menores, antes y después de la introducción de la vacuna contra el rotavirus. Salud Publica Mex. 2009;51:285-90.

14. Lew JF, LeBaron CW, Glass RI, Török T, Griffin PM, Wells JG, Juranek DD, Wahlquist SP. Recommendations for collection of laboratory specimens associated with outbreaks of gastroenteritis. MMWR Recomm Rep. 1990; 39(RR-14):1-13

15. Reingold AL. Outbreak investigations-a perspective. Emerg Infect Dis. 1998;4(1):21-7.

16. Wells GA, Shea B, O'Connell D, Peterson J, Welch V, Losos M, et al. The NewcastleOttawa Scale (NOS) for assessing the quality of nonrandomised studies in meta-analyses. Disponible en: http://www.ohri.ca/programs/ clinical_epidemiology/oxford.asp Acceso el 12 de agosto de 2011

17. Snelling TL, Schultz R, Graham J, Roseby R, Barnes GL, Andrews RM, et al. Rotavirus and the indigenous children of the Australian outback: monovalent vaccine effective in a highburden setting. Clin Infect Dis. 2009;49(3): 428-31.

18. Gelbart B, Hansen-Knarhoi M, Binns P, Krause V. Rotavirus outbreak in a remote Aboriginal community: the burden of disease. J Paediatr Child Health. 2006;42(12):775-80.

19. Kirkwood C, Bogdanovic-Sakran N, Barnes G, Bishop R. Rotavirus serotype G9P[8] and acute gastroenteritis outbreak in children, Northern Australia. Emerg Infect Dis. 2004; 10(9):1593-600.

20. Kelkar SD, Ray PG, Shinde DN. An epidemic of rotavirus diarrhoea in Jawhar Taluk, Thane district, Maharashtra, India, December 2000January 2001. Epidemiol Infect. 2004;132(2): 337-41.

21. Karmakar S, Rathore AS, Kadri SM, Dutt S, Khare S, Lal S. Post-earthquake outbreak of rotavirus gastroenteritis in Kashmir (India): An epidemiological analysis. Public Health. 2008;122(10):981-9.

22. Sugunan AP, Roy S, Murhekar MV, Naik TN, Sehgal SC. Outbreak of rotaviral diarrhoea in a relief camp for tsunami victims at Car
Nicobar Island, India. J Public Health (Oxf). 2007;29(4):449-50.

23. Martinelli D, Prato R, Chironna M, Sallustio A, Caputi G, Conversano $M$, et al. Large outbreak of viral gastroenteritis caused by contaminated drinking water in Apulia, Italy, May-October 2006. Euro Surveill. 2007;12(4).

24. Scarcella C, Carasi S, Cadoria F, Macchi L, Pavan A, Salamana M, et al. An outbreak of viral gastroenteritis linked to municipal water supply, Lombardy, Italy, June 2009. Euro Surveill. 2009;14(29).

25. Bucardo F, Karlsson B, Nordgren J, Paniagua M, González A, Amador JJ, et al. Mutated $\mathrm{G} 4 \mathrm{P}$ [8] rotavirus associated with a nationwide outbreak of gastroenteritis in Nicaragua in 2005. J Clin Microbiol. 2007;45(3):990-7.

26. Guardado JA, Clará W AW, Turcios RM, Fuentes RA, Valencia D, Sandoval R, et al. Rotavirus in El Salvador: an outbreak, surveillance and estimates of disease burden, 20002002. Pediatr Infect Dis J. 2004;23(10 Supl): 156-60.

27. Fischer TK, Ashley D, Kerin T, ReynoldsHedmann E, Gentsch J, Widdowson MA, et al. Rotavirus antigenemia in patients with acute gastroenteritis. J Infect Dis. 2005;192(5):913-9.

28. Corwin AL, Subekti D, Sukri NC, Willy RJ Master J, Priyanto E, et al. A large outbreak of probable rotavirus in Nusa Tenggara Timur, Indonesia. Am J Trop Med Hyg. 2005;72(4): 488-94.

29. Villena $C$, Gabrieli R, Pintó RM, Guix $S$, Donia D, Buonomo E, et al. A large infantile gastroenteritis outbreak in Albania caused by multiple emerging rotavirus genotypes. Epidemiol Infect. 2003;131(3):1105-10.

30. Ford-Jones EL, Wang E, Petric M, Corey P, Moineddin R, Fearon M. Hospitalization for community-acquired, rotavirus-associated diarrhea: a prospective, longitudinal, population-based study during the seasonal outbreak. The Greater Toronto Area/Peel Region PRESI Study Group. Pediatric Rotavirus Epidemiology Study for Immunization. Arch Pediatr Adolesc Med. 2000;154(6):578-85.

31. Amador JJ, Vicari A, Turcios-Ruiz RM Melendez D AC, Malek M, Michel F, et al. Outbreak of rotavirus gastroenteritis with high mortality, Nicaragua, 2005. Rev Panam Salud Publica. 2008;23(4):277-84. 
32. Lee JI, Song MO, Chung JY, Han TH, Ahn YM, Seo JW, et al. Outbreak of rotavirus variant P[8] in Seoul, South Korea. J Med Virol. 2008;80(9):1661-5.

33. Krieg RE, López JA, Jaramillo R, Reyes LG, Duncan JF. An outbreak of rotaviral gastroenteritis in Belize. West Indian Med J. 1992; 41(1 supl):51.

34. Sum MS, Perera D, Ramji N, Elie F, Cardosa MJ. Molecular Characterization of Rotavirus A Associated with Outbreaks of Acute Gastroenteritis in Sarawak in 2001 and 2007. Int J Infect Dis. 2008;12(1 supl):91-2.

35. Palombo EA, Bugg HC, Masendycz PJ, Coulson BS, Barnes GL, Bishop RF. Multiplegene rotavirus reassortants responsible for an outbreak of gastroenteritis in central and northern Australia. J Gen Virol. 1996;77(6):1223-7.

36. Centers for Disease Control and Prevention (CDC). Outbreak of severe rotavirus gastroenteritis among children-Jamaica, 2003. MMWR Morb Mortal Wkly Rep. 2003;52(45):1103-5.

37. World Health Organization. Rotavirus vaccines. Wkly Epidemiol Rec 2007;82:285-96

38. Rotavirus surveillance worldwide-2009. Wkly Epidemiol Rec. 2011:29;86(18):174-6.

39. Santos N, Hoshino Y. Global distribution of rotavirus serotypes/genotypes and its implication for the development and implementation of an effective rotavirus vaccine. Rev Med Virol. 2005;15(1):29-56.
40. Kane EM, Turcios RM, Arvay ML, García S, Bresee JS, Glass RI. The epidemiology of rotavirus diarrhea in Latin America. Anticipating rotavirus vaccines. Rev Panam Salud Publica. 2004;16(6):371-7.

41. Dicker R. Principles of Epidemiology in Public Health Practice. 3rd ed. U.S. Department of Health and Human Services. Atlanta, U.S. CDC; 2006

Manuscrito recibido el 27 de septiembre de 2010. Aceptado para publicación, tras revisión, el 19 de julio de 2011.

ABSTRACT Objective. Determine the epidemiological profile of outbreaks of acute diarrheal disease caused by rotavirus (RV) occurring in pediatric patients, based on a critical review of the literature published between 2000 and 2010.

\section{Diarrheal disease caused by rotavirus in epidemic outbreaks}

Key words Rotavirus; diarrhea, infantile; child health (public health); gastrointestinal diseases; disease outbreaks. 